\title{
Pratiques de Térence : le commentaire des six comédies par Adrien Barlandus, professeur humaniste (Louvain, Rutger Rescius, 1530)
}

\section{Mathieu Ferrand}

\section{CpenEdition}

Journals

Édition électronique

URL : http://journals.openedition.org/rhetorique/565

DOI : $10.4000 /$ rhetorique.565

ISSN : 2270-6909

Éditeur

UGA Éditions/Université Grenoble Alpes

Édition imprimée

ISBN : 978-2-37747-029-7

Référence électronique

Mathieu Ferrand, «Pratiques de Térence : le commentaire des six comédies par Adrien Barlandus, professeur humaniste (Louvain, Rutger Rescius, 1530) », Exercices de rhétorique [En ligne], 10 | 2017 mis en ligne le 26 décembre 2017, consulté le 02 octobre 2020. URL : http://journals.openedition.org/ rhetorique/565; DOI : https://doi.org/10.4000/rhetorique.565

Ce document a été généré automatiquement le 2 octobre 2020.

\section{c) (†) (-)}

Les contenus de la revue Exercices de rhétorique sont mis à disposition selon les termes de la Licence Creative Commons Attribution - Pas d'Utilisation Commerciale - Partage dans les Mêmes Conditions 4.0 International. 


\title{
Pratiques de Térence : le
} commentaire des six comédies par Adrien Barlandus, professeur humaniste (Louvain, Rutger Rescius, 1530)

\author{
Mathieu Ferrand
}

1 Térence n'a jamais cessé d'être lu, pendant tout le Moyen Âge ${ }^{1}$. Au Xve siècle, la redécouverte du commentaire de Donat puis l'invention de l'imprimerie renouvellent l'intérêt pour ses comédies ; autour de 1500, Guy Jouenneaux et Josse Bade publient les premiers commentaires modernes du poète comique, à Lyon puis Paris ${ }^{2}$. D'autres humanistes suivent leur exemple, tel Petrus Marsus à Strasbourg, ou Adrien Barlandus à Louvain ${ }^{3}$.

2 C'est au travail de ce dernier que sera consacré le présent article ${ }^{4}$. Son texte, publié en 1530, a en effet un intérêt tout particulier. Adrien Barlandus est tout d'abord un proche d'Érasme qu'il ne cesse de citer et de louer dans ses écrits ${ }^{5}$ : son commentaire des six comédies se fait l'écho des réflexions du maitre de Rotterdam sur les textes antiques, le théâtre ou la pédagogie nouvelle. Par ailleurs, Barlandus est essentiellement un praticien de l'éducation. Contrairement à Josse Bade, par exemple, qui s'est surtout illustré comme imprimeur humaniste, Barlandus n'a jamais cessé d'enseigner dans l'université de Louvain. Il a laissé une œuvre abondante qui témoigne de sa pratique pédagogique quotidienne. Aussi proposons-nous d'interroger l'inscription de son commentaire dans cette pratique. De quels usages de Térence le volume publié est-il ou porte-t-il la trace? 


\section{Les pièces liminaires}

3 Élève puis professeur au collège du Porc, l'une des quatre pédagogies de l'université louvaniste ${ }^{6}$, Adrien Barlandus (1486-1538) fut le premier professeur de latin du Collège trilingue, avant d'enseigner comme précepteur privé puis d'obtenir la charge prestigieuse de rhetor publicus. Éditeur de textes anciens (Tite-Live, Pline, Virgile, etc.), il publia plusieurs manuels scolaires à l'attention de ses étudiants : recueils d'adages, de colloques, précis de rhétorique. En 1530, c'est un homme dont la réputation en matière d'enseignement et d'érudition est bien établie qui publie à Louvain le volume des six comédies.

\section{Le livre et ses destinataires}

4 Le livre parut sous les presses de Rutger Ressen (Rescius) ${ }^{7}$. Professeur de grec au Collège trilingue, il avait pris un an plus tôt la suite de l'imprimeur Thierry Martens, premier éditeur de Barlandus ${ }^{8}$. Son ambition était de fournir aux étudiants louvanistes des textes de qualité, à un prix abordable 9 .

5 Aussi les deux hommes choisirent-ils un format in quarto, plus maniable et moins cher que les incunables in-folio. Barlandus lui-même explique ce choix dans une lettre « aux maitres d'école du Brabant, de Flandre et de Hollande ", placée en tête de son édition de Pline :

Curavi festivissimas quasque C. Plinii epistolas cum scholiis meis forma portabili excudendas, ut ab omnibus et interpretibus et scholasticis emi quam minimo possent ${ }^{10}$.

J'ai veillé à faire imprimer les plus charmantes lettres de Pline avec mes commentaires, sous une forme portative, afin qu'elles puissent être achetées au moindre prix par tous ceux qui l'expliquent et par tous ceux qui l'étudient.

6 Le paratexte du commentaire de Térence insiste lui aussi sur l'existence d'un double lectorat. Un court avis « à l'étudiant » («studioso lectori ») ouvre ainsi l'ouvrage ; celui-ci fut publié «afin que les jeunes gens apprennent le profit que l'on peut tirer de la lecture des comédies ${ }^{11}$ ». En fin de volume, une épître est adressée " aux jeunes élèves de Louvain » («Ad Lovanienses Tyrunculos ») :

Hos commentarios qui dedit, sperat se vobis rem non ingratam fecisse, iuvenes optimi. Scripsit omnibus scholis, sed maxime quatuor huius Academiae celebrioribus collegiis ${ }^{12}$.

Celui qui a donné ce commentaire espère avoir fait une œuvre qui vous sera agréable, excellents jeunes gens. Il a écrit pour toutes les écoles, mais surtout pour les quatre collèges si fréquentés de notre Université.

Le premier dédicataire du volume n'en est pas moins Jean de Fevyn, ancien camarade d'étude, écolâtre du chapitre de Saint-Donatien, ami de Thomas More et d'Érasme; Barlandus lui adresse une longue lettre liminaire ${ }^{13}$.

7 La présence de ce double lectorat - les étudiants et le collègue savant - est caractéristique de ces textes scolaires qui prévoient (au moins) deux types de réceptions, l'une directe - celle de professeurs ou d'érudits, capables de faire un usage personnel de la matière rassemblée - et l'autre indirecte - celle d'étudiants et de jeunes élèves qui, certes, peuvent se procurer le volume et le lire, mais ont aussi besoin d'un maitre pour en apprécier la richesse. Si les élèves sont idéalement les vrais destinataires d'une telle publication, car c'est pour la jeunesse que travaille Barlandus, l'objet lui-même a plus de chance d'être goûté dans toute son épaisseur par des hommes éclairés tels que Fevyn ${ }^{14}$. 
8 La genèse même du volume, telle que la présente la lettre dédicatoire, confirme ce double horizon de lecture. Le propos offre du reste un témoignage précieux sur l'articulation du cours réellement donné et du commentaire imprimé :

Ante annos aliquammultos quum Louanii publice Terentium enarrarem, Augustinus quidam Reymarius, [...] meus auditor [...] me praelegente non pauca excepit, non pauca ex nostris autographis descripsit. Ex veteribus quoque grammaticis non parum multa collegit, quae postea ego ad manus perlata relegi, castigavi, et nonullis tanquam verrucis deformibus recisis in unum corpus restitui. Utque iustus esset commentarius adieci figuras, adieci quae ad consilium et oratorium pertinerent artificium, sperans fore ut per huius poetae sic enarrati publicationem aliqua ex parte eloquentia in antiquam illam, e qua deiecta [323] est, dignitatem restitueretur, et quae ante annos haud ita multos, Italiae tantum finibus continebatur, nunc brevi tempore in nullas non totius orbis partes perveniret ${ }^{15}$.

Comme, à Louvain, il y déjà assez longtemps, j'expliquais Térence en public, un certain Augustin Reymarius, [...] l'un de mes étudiants, [...] prit des notes abondantes pendant mes cours et abondamment les compléta, à partir de mes propres notes autographes. Il recueillit aussi de nombreux éléments chez les grammairiens anciens que j'ai, par la suite, reportés de mes propres mains, corrigés et rassemblés en un seul volume, après les avoir débarrassés pour ainsi dire de quelques laides verrues. Pour que mon commentaire soit tout à fait convenable, $j$ 'ai ajouté des figures, j'ai ajouté ce qui pouvait être utile à la formation du jugement et du discours, espérant rendre, en publiant une telle édition commentée de notre auteur, une partie de son éloquence à cette ancienne dignité dont il fut privé, et qu'ainsi, ce qui, il y a peu d'années encore, était contenu dans les seules limites de l'Italie, à présent se répande, en peu de temps, dans toutes les contrées de la terre.

Le point de départ est donc le cours dispensé jadis par Barlandus dont la mémoire fut conservée par les notes de l'étudiant et par celles du professeur. L'élève a mis au net cette matière brute et a ajouté des gloses tirées des meilleurs grammairiens. Puis le professeur a repris, recopié et corrigé le tout et a fait de nouveaux ajouts. Ainsi l'objet publié se compose-t-il de différentes strates: au souvenir du cours réellement donné par le grammaticus - devant sans doute de jeunes élèves - s'ajoutent des gloses savantes, d'abord grammaticales, puis rhétoriques - ce sont celles du rhetor. Le volume publié témoigne d'une sédimentation de ces savoirs, que rien ne distingue plus sur le plan formel. Chacun, selon son niveau, peut y trouver matière à réflexion.

\section{Prolégomènes}

9 Dans le De ratione studii, Érasme donne l'exemple d'un commentaire mené selon ses vœux, qui s'appuie précisément sur une comédie de Térence ${ }^{16}$. La lectio se déploie ainsi en quatre temps: les prolégomènes sont de nature encomiastique et portent sur l'auteur et sur le genre; l'explication de détail rend compte de la construction de l'intrigue, de la versification, de la syntaxe, du vocabulaire et enfin, des procédés rhétoriques; le texte étudié est ensuite rapproché d'autres textes susceptibles de l'éclairer et d'ouvrir le champ de la réflexion. Enfin, la fable se prête à une interprétation d'ordre philosophique et éthique. L'explication forme un tout organique et présente une progression hiérarchisée ; Érasme pense ainsi l'articulation de tous les niveaux des apprentissages. Que retient le commentaire imprimé - analytique, selon la tradition - de l'approche synthétique du Rotterdamois?

10 L'accessus ad auctorem se lit dans les prolégomènes. C'est de fait l'une des fonctions des épîtres que de faire l'éloge de Térence et de ses comédies ${ }^{17}$. Barlandus s'amuse par exemple de ce « passage obligé » dans la lettre à Fevyn : 
In more esse video non paucis interpretatibus ut eum quem interpretantur autorem, uberius laudent, ac longiore prosequantur encomio. Ego de Terentio tantum dicam non alium non esse scriptorem, qui loqui elegantius doceat. Quod vero ad argumentum pertinet, [...] personarum colloquiis tamquam in speculo hominum mores et vitam inspiciendam proponit. Improbe luxuriantes adolescentes coercet, saeviusque increpat ac moderatur. Peccantes verbo correptionis castigat et sermonibus doctrinae salutaris incitat ad probitatem ${ }^{18}$.

Je vois qu'il est dans l'habitude de nombreux commentateurs de louer trop abondamment celui qu'ils commentent et de lui adresser des éloges trop longs. Pour ma part, sur Térence, je dirai seulement que nul autre écrivain n'enseigne à parler avec plus d'élégance ${ }^{19}$. Pour ce qui relève du contenu, [...] il donne à voir les mœurs et la vie des hommes comme en un miroir ${ }^{20}$. Il corrige les jeunes gens qui s'adonnent honteusement aux excès, les blâme fort sévèrement et les modère. Il châtie ceux qui fautent par des mots de reproche et les pousse à la probité par des propos d'une salutaire doctrine.

L'éloge s'articule ici comme ailleurs autour de deux piliers, qui distinguent la forme et le fond : le profit moral - il faut défendre Térence contre le soupçon d'immoralité - et la formation de l'éloquence, au sens large puisqu'il s'agit essentiellement - mais pas seulement, nous le verrons - d'apprendre par la lecture des comédies à parler le sermo quotidianus, la langue des échanges quotidiens.

11 Si l'éloge est bien présent, les liminaires n'apportent pourtant pas de connaissance détaillée sur l'auteur lui-même - sa vie - ou sur le genre comique. On sait que beaucoup d'éditions de Térence au XVI ${ }^{\mathrm{e}}$ siècle s'ouvraient sur le De comoedia et Tragoedia de Donat, lorsque les humanistes eux-mêmes ne rédigeaient pas leurs propres textes. Barlandus, lui, n'a pas ici de telles ambitions ${ }^{21}$. En revanche le paratexte de Barlandus présente un résumé détaillé des six pièces ${ }^{22}$ puis une énumération des leçons morales qu'elles contiennent $^{23}$. Ainsi, avant même la lecture du corpus, le lecteur peut se familiariser avec des intrigues toujours complexes et jugées édifiantes. Barlandus a par ailleurs ajouté un extrait du De copia d'Érasme, qu'il intitule « Varietas personarum in Comoediis ex commentario Erasmi de rerum copia ${ }^{24}$ "; ces quelques lignes insistent sur les contrastes entre les personnages de même type, dans chaque comédie ${ }^{25}$. Le lecteur est donc invité à entrer par paliers au cœur même de l'œuvre, dont la savante économie et la sage moralité sont ainsi soulignées. S'ajoutent à ces liminaires d'autres éléments du péritexte : des rubriques marginales accompagnent les gloses et soulignent certains points importants, qui se trouvent recensés dans l'index final. Un tel dispositif permet une circulation libre dans ce texte copieux : le volume rend donc possible différents types de lectures, linéaire ou buissonnière, centrifuge (à partir du texte de Térence) ou centripète (à partir de notions, de savoirs que l'index permet de retrouver dans les comédies).

\section{Le texte et ses gloses}

12 Le corps même de l'ouvrage présente le texte de Térence, divisé en actes et scènes, et ses gloses. Chaque scène est suivie d'un argumentum, qui en résume le contenu, puis de son commentaire, en caractères plus petits. Des marges permettent les annotations manuscrites. 


\section{Le grammaticus}

13 Dans l'étude qu'il consacra jadis à Adrien Barlandus, Etienne Daxhelet a montré que le commentateur de Térence utilisait comme texte de base celui de l'édition de Robert Estienne parue à Paris en 1529. Si, en général, il le suit assez fidèlement, il corrige cependant quelques leçons ${ }^{26}$. Pour ce faire, il utilise, outre les gloses philologiques de certains de ses devanciers ou contemporains, une édition aldine et un manuscrit gantois aujourd'hui perdu dont il est question à plusieurs reprises dans le volume; au détour d'une note philologique, Barlandus raconte ainsi, en un récit circonstancié, l'usage qu'il en fit ${ }^{27}$. Mais il n'insiste que fort ponctuellement sur ce travail-là et n'en dit presque rien dans ses liminaires : il préfère alors, à la persona du philologue, celle du professeur $^{28}$.

14 Au reste, son ambition est moins de corriger et d'établir les textes que de les donner à lire et à comprendre aux étudiants. C'est en ce seul nom qu'il se permet de citer et amender les commentaires de ses devanciers :

Omnes bonarum artium cultores oro, ne laborem meum reprehensionem existiment aliorum, qui ante me scripserunt in hunc poetam. Ad iuvanda studia offert unusquisque quod potest. [...] In primis operam dedimus, ut ab aliis dicta obscurius, plano sermone et lucido exponeremus, ad erudiendam posteritatis infantiam ${ }^{29}$.

Je prie pour que nul de ceux qui cultivent les bonnes lettres n'aille penser que mon travail remet en cause les travaux de ceux qui avant moi ont écrit sur ce poète. Chacun apporte ce qu'il peut au progrès des études. [...] Avant tout, nous avons voulu expliquer ce que d'autres ont dit de façon trop obscure en un propos clair et limpide ${ }^{30}$, afin d'instruire la jeunesse de demain.

15 Ainsi, le travail d'explanatio porte aussi bien sur le texte de Térence que sur les commentaires antérieurs. L'édition d'Estienne contenait en particulier les gloses de Donat sur cinq des six comédies et celles de Calphurnius sur l'Heautontimoroumenos. Elles constituent de fait la source principale de Barlandus. Donat, en particulier, se trouve cité à chaque page. Bien loin toutefois de tout retenir, Barlandus sélectionne et discute parfois la pertinence de ses commentaires: "Non mihi probatur quod Donatus scripsit $^{31}$ [...]. » De façon générale, à l'égard des autorités citées, il n'hésite pas à prendre position et à les éclairer de commentaires divers, les siens ou ceux d'autres figures autorisées $^{32}$. In fine, son texte est bien plus riche que le commentaire de Donat: il le complète selon une logique amplificatrice, qui intègre d'autres autorités, prosateurs et poètes. Le commentaire, conformément aux habitudes du temps, se construit comme une polyphonie, voire une œuvre collective mais critique, et, à cet égard, rappelle ses liens avec la pratique scolaire.

16 Comme le préconise Érasme, mais comme le faisait déjà Donat, le premier niveau de l'analyse propose une élucidation du sens littéral de certains vers, de certaines expressions. C'est ainsi que dans la première scène de L'Andrienne, le grammaticus, professeur des petites classes, commente :

PAUCIS TE VOLO.) Colloqui et paucis verbis tecum agere volo ${ }^{33}$.

DEUX MOTS!) Je veux te parler et m'entretenir avec toi en deux mots.

17 La reformulation est inspirée du commentaire plus synthétique de Donat, qui ajoutait une notation technique non retenue par Barlandus: «Et est figura $\sigma \dot{v} \lambda \lambda \eta \mu \psi \iota \varsigma$ [sullempsis $]^{34} »$. Mais alors que Donat s'en tenait là, Barlandus imagine la question d'un interlocuteur. Il peut ainsi glisser du simple développement d'une formule elliptique au commentaire véritable : 
Sed cur dicit "paucis te volo", cum satis prolixe cum eo loquuturus sit? Dic simulare eum paucis commentaturum sese cum liberto, ut spe brevitatis audiatur attentius ${ }^{35}$.

Mais pourquoi dit-il « Deux mots ! » alors qu'il s'apprête à s'entretenir avec lui assez longuement? Disons qu'il feint de vouloir parler en deux mots avec l'affranchi, afin de le rendre, en promettant d'être bref, plus attentif.

Au-delà de l'intérêt qu'il présente du point de vue de l'explanatio, ce commentaire dit quelque chose de la pragmatique du commentaire. Il emprunte, en effet, à l'échange à bâtons rompus tel qu'il pouvait s'entendre dans les classes : le commentaire accueille une parole seconde qui permet au commentateur d'aller plus loin dans ces explications et de passer de la littera au sensus.

Évidemment, l'élucidation du sens littéral s'appuie très souvent sur des explications d'ordre grammatical. Celles-ci permettent non seulement de comprendre le sens, mais aussi de comprendre le fonctionnement même de la langue latine. Le commentaire renvoie parfois au seul usage, et prend alors des exemples qui parlent à chacun, et notamment aux élèves :

Quando per non interrogamus, non id agitur ut respondeas nescienti, sed ut cogaris assentiri scienti, ut quum dicam "Non commodavi tibi codicem?", volo per hunc loquendi modum te cogere ut mihi id scienti assentiaris ${ }^{36}$.

Quand nous interrogeons avec la négation, nous ne l'ajoutons pas pour que l'on nous réponde comme à des ignorants, mais pour que l'on confirme ce que l'on sait, comme si je disais «Ne t'ai-je pas prêté mon livre?»: je veux, en parlant de cette façon, t'obliger à admettre ce que je sais.

Là encore, on entend le maître en classe, s'adressant à de jeunes gens ${ }^{37}$. Plus souvent, les commentaires grammaticaux se font techniques, et s'appuient sur l'autorité des grammairiens, au premier rang desquels Priscien, très abondamment cité, mais aussi Perotti, chez les modernes, et d'autres encore. Les commentaires sont alors de double nature : soit ils portent sur un cas précis, qu'il s'agit seulement d'élucider, soit ils se développent en véritables leçons de grammaire, parfaitement autonomes ${ }^{38}$.

Outre ses connaissances grammaticales, le grammaticus livre, comme il se doit, des renseignements sur les realia antiques qui permettent eux aussi d'éclairer le sensus. Les sources sont, en cette matière encore, fort diverses, mais certains contemporains sont privilégiés tels Lorenzo Valla ou Guillaume Budé ${ }^{39}$. Barlandus cite par exemple, à propos des Satrapes (f. G4v), ses Annotations sur les Pandectes ou encore le De asse quand il s'agit de convertir les monnaies anciennes en devises modernes ${ }^{40}$. De tels rapprochements avec la civilisation contemporaine ne sont pas nombreux mais toujours pittoresques; ils apportent, dans la lecture parfois fastidieuse du commentaire, une touche qui charme le lecteur. Ils ont pour vertu, aussi, d'abolir le temps. Ainsi Barlandus compare-t-il le pistrinum aux moulins modernes de Louvain et des villes brabançonnes (f. D2v), les plateae antiques à la grand-rue de Malines, « ville aujourd'hui dirigée par la très illustre Princesse Marguerite, tante de l'Empereur Charles » (« cui nunc imperat urbi illustrissima Domina Margareta Caroli Caesaris amita », $\mathrm{f}$. L4r) ; il cite, à propos du salut de Chrémès dans L'Andrienne (f. H3r-v), une lettre d'Érasme à Fausto Andrelini sur cette étrange façon qu'ont les Anglais de s'embrasser en toute occasion.

De cette manière, Barlandus parvient à rapprocher le monde ancien du monde familier de ses lecteurs ${ }^{41}$. L'enjeu est important car ce rapprochement permet d'abstraire, de l'action représentée, les enseignements moraux que promettaient les pièces liminaires. De fait, si, la comédie est, comme Barlandus le rappelle après Donat, un «miroir des mœurs ", sa vertu morale se fonde sur ce qu'elle dit encore aux contemporains de 
Barlandus, au-delà des contingences historiques. Le succès scolaire de Térence se fonde notamment sur l'identification possible des jeunes élèves avec ses héros, fils rebelles, qui préfèrent à l'autorité du pédagogue les plaisirs de l'amour, de la boisson, etc. C'est parfois l'argument qui, en tête de scène, souligne l'enjeu moral du propos :

Hic docet Terentius immoderatum vini usum fugiendum esse iis qui seriis praesunt negotiis ${ }^{42}$.

Ici Térence nous enseigne qu'il faut fuir tout usage immodéré du vin quand on est en charge d'une affaire sérieuse.

Plus souvent c'est dans le corps du commentaire que surgit la leçon. Ici, le professeur condamne le mariage ${ }^{43}$. Là, il propose une distinction fondamentale :

Hoc tempore obsequium amicos, veritas odium parit.) Proverbium hoc non admonet quid oporteat fieri, sed quid vulgo fiat ostendit ${ }^{44}$.

De nos jours, la complaisance procure des amis, et la vérité la haine.) Ce proverbe ne nous indique pas ce qu'il convient de faire, mais nous montre ce qui se produit généralement.

Mettant en garde contre toute lecture littérale, Barlandus cite ici l'adage II, 9, 53 d'Érasme, et son long commentaire. De fait, les Adages sont, en matière de lecture morale, la référence récurrente du professeur: puisant abondamment dans les comédies de Térence, le recueil permet d'arracher l'énoncé à son ancrage particulier et à son sens immédiat (sensus) pour en tirer des enseignements (sententia) que Barlandus se plaît à citer, presque à chaque page ${ }^{45}$. Rappelons que Barlandus avait précisément publié un épitomé des Adages érasmiens.

\section{Le rhetor}

21 M. T. Herrick a montré, dans son étude déjà ancienne, la prégnance du modèle rhétorique dans les commentaires humanistes des comédies antiques ${ }^{46}$. Térence est, pour Barlandus comme pour tous ses contemporains, un maitre du discours, non seulement du sermo quotidianus, mais aussi, plus étonnamment, du discours formel, de l' oratio. À cet égard, il permet de former les latinistes débutants, élèves du grammaticus, ainsi que les étudiants des classes de rhétorique.

Au niveau micro-structurel, le rhetor, conformément à ce qu'il annonçait dans l'extrait déjà cité de sa lettre préface, identifie les figures ou les lieux du discours :

Effundite, emite, facite.) Figura est, qua nos invitos aliquid permittere ostendimus. Cicero permissionem vocat ${ }^{47}$.

Dépensez, achetez, faites donc !). Il s'agit de la figure par laquelle nous montrons que nous permettons une chose sans la souhaiter. Cicéron l'appelle " permission ».

La définition de la figure se trouve chez Donat qui donne son nom grec. Mais la mention de Cicéron, qui propose un équivalent latin, est le fait de Barlandus : il ne suffit pas de nommer les figures et d'évoquer leurs effets; il faut aussi nourrir le commentaire de références savantes qui autorisent le propos.

Au-delà de l'inventio et de l'elocutio, le maître est attentif à la dispositio. Or, pour rendre compte de la construction d'une pièce, ou d'une unité de discours, il lui faut surmonter la fragmentation propre au commentaire linéaire ${ }^{48}$. De fait, comme l'écrit E.W. Robbins, «comme il s'appuie assez fortement sur Donat, le commentaire de Barlandus porte plus sur le choix des mots que sur des événements de plus d'étendue ${ }^{49}$ ». Certes, Donat n'est pas seul responsable ; bien souvent, le grammairien se montre même plus attentif à la dynamique de certaines scènes, ou à leur fonction dans l'économie de la pièce, que le professeur louvaniste ${ }^{50}$. Au reste, celui-ci ne s'interdit pas, 
parfois, de porter un regard d'ensemble sur l'œuvre qu'il commente, ou sur certains passages: c'est notamment la fonction des seuils - résumés liminaires du volume, argumenta, premières lignes de certains commentaires ${ }^{51}$ - mais aussi, parfois, le rôle de citations plus longues, qui permettent d'insérer dans le commentaire vers à vers des analyses d'une autre nature. Ainsi reproduit-il in extenso un extrait du De inventione où Cicéron montre comment, dans la scène 1 de L'Andrienne, aux vers 49 et suivants, le propos du vieux père est conforme au plan qu'il avait d'abord annoncés2. C'est là un excellent exemple à suivre pour la construction de tout discours. Plus loin dans la même scène, le professeur recourt à l'autorité des modernes, et précisément à celle de Rodolphe Agricola :

Interea mulier quaedam.) Huius loci artificium Rodolphus Agricola usque ad miraculum doctus, nec minus etiam facundus homo in suis libris de inventione dialectica ostendit, cuius verba non gravabor in hoc commentarium ascribere: "Illud, inquit, videtur fere observatum, ut undecunque petitum semper ponant favorabilem primum narrationis ingressum et ab aliquo, quod causam suam commendet, auspicentur ${ }^{53}[. .]$.$» .$

Sur ces entrefaites une femme...) Rodolphe Agricola, homme merveilleusement savant et non moins éloquent, montre l'habileté de ce passage dans son livre De l'Invention dialectique. Je n'hésite pas à citer ses mots dans ce commentaire : «C'est, dit-il, une loi non écrite, semble-t-il, de toujours formuler le début de l'exposition des faits, quel qu'en soit le point de départ, d'une manière engageante et de débuter par un point qui servira à son affaire [...]. »

Agricola, de fait, développe sur plus d'une page une analyse des moyens par lesquels le père parvient à minimiser les torts de son fils. Le sermo quotidianus devient ici modèle d' oratio dont la dispositio est étudiée du point de vue de ses effets, sans qu'aucun élément hétérogène - remarque grammaticale, glose sur les realia, etc. - ne vienne en interrompre le cours. Tout en s'inscrivant dans la tradition du commentaire linéaire, qui recourt abondamment à la citation, Barlandus trouve donc le moyen d'accueillir une autre manière d'expliquer les textes, affranchie des contraintes du genre. Une telle pratique pouvait être celle des classes de rhétorique et trouve ainsi sa place dans le volume $^{54}$.

La memoria n'est guère abordée dans le commentaire - elle était pourtant au cœur de la pédagogie du temps et Barlandus a rapporté ailleurs la façon dont ses élèves apprenaient par cœur certaines pièces de Térence ${ }^{55}$. L'actio, en revanche, fait l'objet de remarques nombreuses. De fait, le professeur semble avoir porté à la question un intérêt tout particulier. Au cœur du volume, juste avant le prologue des Adelphes, il a publié un prologue qu'il a lui-même composé à l'occasion, nous dit-on, d'une mise en scène de ce texte par ses étudiants ${ }^{56}$. Le commentateur de Térence était aussi, de fait, un praticien du théatre et nous conservons de lui quatre autres prologues originaux, pour divers spectacles ${ }^{57}$. Aussi était-il sensible aux rapports étroits que Cicéron et Quintilien, déjà, soulignaient entre jeu d'acteur et actio oratoire.

Barlandus n'oublie pas la vocation scénique des textes qu'il commente. Dans ses gloses sur L'Andrienne, il évoque en ces termes la pratique du salut :

Antiquis mos erat, ut amicus amicum non simpliciter, sed osculo et complexu salutaret, neque id solum apud Iudaeos, verum etiam apud Graecos et Romanos. Quare qui recte volent agere fabulas, sive graecas, sive latinas, ubi locus exiget, ipsi quoque advenientem amicum osculo et complexu salutabunt, qui mos hodie servatur in Anglia ${ }^{58}$.

Les Anciens avaient pour habitude, de ne pas simplement se saluer, mais de se donner un baiser et de se prendre dans les bras, non seulement chez les Juifs, mais aussi chez les Grecs et les Romains. Raisons pour laquelle ceux qui voudront jouer les pièces, soit en grec, soit en latin, quand le passage l'exigera, salueront eux- 
mêmes l'ami qui vient à eux en lui donnant un baiser et en l'embrassant, habitude que l'on observe aujourd'hui encore en Angleterre.

Imaginant la mise en scène d'autres textes, latins - témoignage rare - et grecs témoignage exceptionnel -, le commentateur se fait ici dramaturge. Son texte nous révèle en effet les principes qui, selon ses vœux, doivent présider au jeu de l'acteur : il lui faut, autant que possible, se conformer aux pratiques antiques qu'il s'agit de restituer (à la lumière, le cas échéant, de pratiques contemporaines comparables).

Barlandus a-t-il tenté, toutefois, de donner forme scénique à l'idée selon laquelle Calliopius, dont le nom paraît à la fin de L'Andrienne, était le récitant d'un spectacle que les acteurs, en scène, se contentaient de mimer ? Certes, il la reprend, en citant Érasme qui, pour une fois, l'égare ${ }^{59}$, mais nous peinons à imaginer qu'un dispositif si éloigné des pratiques dramatiques contemporaines ait été adopté par le professeur. Du moins, rien ne le laisse penser à la lecture de ses gloses. Les unes font allusion à la pronuntiatio du vers, dans le cadre, peut-être, d'un exercice de lecture ou de déclamation en classe :

Ego propter illam.) Magna uis est in pronominibus. Praeceptores igitur videant ne praecipitanter, sed interrupte haec proferant iuuenes ${ }^{60}$.

Moi, pour elle.) Il y a beaucoup de force dans les pronoms. Les professeurs doivent donc veiller à ce que les jeunes gens profèrent ces mots sans trop de hâte, mais en marquant une pause.

Il campe à cette occasion le professeur au travail ${ }^{61}$. D'autres remarques, nombreuses, évoquent de véritables jeux scéniques. Ainsi, dans Phormion, Barlandus commente les trois premiers mots de la scène 2 de l'acte II :

ITA ne patris.) Si agenda est fabula, Phormio ebrius, ructans et potu satur exeat ${ }^{62}$.

Ainsi de son père.) Si l'on doit jouer la pièce, que Phormion entre en scène en état d'ivresse, éructant et imbibé de boisson.

«Si agenda est fabula ": l'originalité de son propos se trouve précisément dans ce type de formules - au demeurant assez rares. Elle n'est pas, en revanche, dans le contenu même des commentaires, qui, le plus souvent, reprennent les gloses de Donat - c'est le cas dans les deux exemples précédents. Certes, Barlandus ne s'interdit pas alors d'adapter les indications scéniques, avec parfois une certaine pertinence. Dans l'argument de la scène 3 de l'acte IV de L'Andrienne, il met ainsi en évidence une importante remarque de Donat (« consistit haec scena in gestu magis, quam in oratione $\left.e^{63} »\right)$, qui se trouvait mêlée aux autres commentaires, puis il explicite, dans le cours de la scène, certaines gloses, rendant ainsi l'action scénique plus lisible. Pour le reste, Barlandus n'apporte souvent rien de nouveau, sauf lorsque d'autres textes tels que l' Institution oratoire de Quintilien peuvent être cités avec autorité6 ${ }^{64}$. Il supprime ailleurs des commentaires dont l'intérêt pour une éventuelle mise en scène semblait manifeste.

Le volume publié par Adrien Barlandus ne saurait, in fine, être un manuel sur l'actio dramatique; il n'illustre pas une vision personnelle du jeu de scène pas plus qu'une interprétation nouvelle du texte de Térence, mais se veut simplement le recueil de savoirs accumulés et sélectionnés, dans une intention érudite. Quoi qu'il en soit, en reproduisant, au cœur du volume, le prologue qu'il a lui-même rédigé pour les Adelphes, et en faisant quelques allusions aux professeurs qui, dans leur classe, pratiquent la déclamation et le théâtre, Barlandus a souligné avec force la dimension dramatique du texte de Térence, qui avait été longtemps négligée ${ }^{65}$. Il rappelle ainsi que les collèges humanistes ont été des agents décisifs de la renaissance scénique du théâtre, dès les premières années du XVI ${ }^{\mathrm{e}}$ siècle. 


\section{Conclusion. Le livre et la parole vive}

30 Nous souhaitions, dans le présent article, poser la question du rapport du commentaire avec les pratiques vivantes du texte, telles qu'elles existaient dans les collèges au temps d'Érasme. Le commentaire de Barlandus, né d'un cours, porte en lui des traces d'oralité et d'interaction scolaire, comme bien d'autres commentaires contemporains. Mais il n'en est pas l'équivalent écrit, ni même le miroir déformant. Offrant aux étudiants, par rapport à la lectio du grammaticus, une matière considérablement enrichie, il propose aussi une matière à la fois trop copieuse et trop fragmentée, voire fragmentaire, à laquelle seul un professeur éclairé peut donner forme. Car rien ne saurait remplacer la voix du maitre. C'est ce que rappelle l'un des personnages des Dialogi que Barlandus publie en 1524 :

Loquuntur quidem libri nobis, sed dubitandibus non satis commode respondent, deinde altius insident animo, ac haerent tenacius, quae audieris quam quae legeris et tu ipse nos docuisti, ut quoties vivae vocis data esset copia, libentius aucultaremus, quam legeremus ${ }^{66}$. Assurément, les livres nous parlent, mais ils ne répondent pas de façon satisfaisante à celui qui s'interroge ${ }^{67}$. Ensuite, ce que l'on entend pénètre plus profondément dans nos esprits et s'y attache plus solidement que ce que l'on lit et toi-même tu nous as enseigné de telle sorte que, à chaque fois que tu nous as dispensé ta vive parole, nous écoutions plus volontiers que nous ne lisions.

31 Le propos souligne l'incomplétude ontologique du commentaire publié, puisque le livre demeure "un maître muet». Ainsi, faire l'histoire des commentaires scolaires de Térence, à la Renaissance, est à la fois essentiel et insuffisant pour écrire l'histoire de sa réception dans les classes : les volumes conservés ne sont que les traces d'une pratique de Térence autrement plus riche, dont ils se nourrissent et qu'ils alimentent, mais dont ils ne sauraient à eux seuls témoigner. Le commentaire prend sens au sein d'une constellation d'exercices qui, depuis la lectio en classe jusqu'à la mise en scène, en passant par l'apprentissage par cœur ou l'imitatio, rendent pleinement compte de l'étonnante vitalité du théâtre antique, dans les mondes scolaires et universitaires de la première modernité.

\section{NOTES}

1. Sur la réception de Térence dans la longue durée, voir par exemple les articles réunis dans Terence between Late Antiquity and the Age of Printing, éd. G. Torello-Hill et A. J. Turner, Leyde/ Boston, Brill, 2015.

2. H. W. Lawton, Térence en France au XVI e siècle, Paris, Jouve et Cie, 1926, 2 vol. [Slatkine Reprints, 1970] ; M. T. Herrick, Comic theory in the Sixteenth Century, Urbana, University of Illinois Press, 1964 ; P. White, Jodocus Badius Ascensius. Commentary, Commerce and Print in the Renaissance, Oxford, Oxford University Press, 2013. Sur Josse Bade, voir aussi, dans le présent volume, la contribution de Laure Hermand.

3. Pour une vue d'ensemble sur les premiers commentaires humanistes des comédies de Térence, cf. J. Bloemendal, «In the shadow of Donatus : Observations on Terence and some of his early 
modern commentators", Neo-Latin Commentaries and the Management of Knowledge in the Late Middle Ages and the Early Modern Period (1400 -1700), éd. K. Enenkel et H. Nellen, Louvain, Leuven University Press, 2013, p. 295-323 et H. B. Norland, Drama in Early Tudor Britain, 1485-1558, LincolnLondon, University of Nebraska Press, 1995 (en part. p. 65-83).

4. Sur A. Barlandus, voir E. Daxhelet, Adrien Barlandus, humaniste belge, Louvain, Librairie universitaire, 1938.

5. Sur les relations entre Barlandus et Érasme, $c f$. M. Verweij, «The correspondance of Erasmus and Hadrianus Barlandus", Syntagmatia. Essays on Neo-Latin Literature in Honour of Monique MundDopchie and Gilbert Tournoy, éd. D. Sacré et J. Papy, Louvain, Leuven University Press, 2009, p. 233-250.

6. Les pédagogies sont ce qu'on appelle ailleurs des collèges d'exercice : elles offrent le gîte et le couvert, mais aussi l'essentiel des enseignements de la faculté des arts. Sur l'université louvaniste au temps d'Érasme, voir l'ouvrage classique de H. de Vocht, History of the Foundation and the Rise of the Collegium trilingue, Louvain, University Publications, 1951-1953.

7. P. Terentii sex comoediae ex diversis antiquis exemplaribus emendatae, cum non vulgaribus commentariis eruditissimi viri Adriani Barlandi, Rhetoris inclytae Academiae Lovaniensis. In quibus et artificium ostenditur oratorium et multi difficiles Poetae nodi explicantur, quos interpretes alii intactos reliquerant, Louvain, Rutger Rescius, 1530. Pour une première approche de ce volume, voir les pages que lui consacre E. Daxhelet, op. cit., p.77-82. E. Daxhelet publie par ailleurs un grand nombre des lettres conservées de Barlandus, dont ses épîtres liminaires (ibid., p. 237-331). Lorsque, dans les pages qui suivent, je cite l'une de ces pièces liminaires, j'indique en note les folios de l'édition originale, puis le numéro que lui attribue Daxhelet et les pages de l'ouvrage où il la reproduit.

8. Ibid., p. 321.

9. La publication $d u$ texte, sans même parler du commentaire, est alors déjà un progrès considérable : avant elle, le maître devait dicter le texte étudié à ses étudiants, ce qui était une perte de temps et source de nombreuses erreurs. Cf. le propos liminaire de Barlandus à son édition des Complures Luciani dialogi a Desiderio Erasmo Roterodamo uiro utriusque linguae doctissimo in latinum conuersi (Louvain, Thierry Martens, 1512) : le professeur fit publier le texte de Lucien après s'être rendu compte que les notes prises par ses étudiants sous sa dictée étaient illisibles (E. Daxhelet, op.cit., p. 244-245). M. Furno rapporte un témoignage semblable à propos de Mathurin Cordier, professeur parisien et éditeur des Distica Catonis («Création d'ateliers, affaire de famille? Les éditions de Térence sur les presses des Estienne (1526-1551) », Créations d'atelier. L'éditeur et la fabrique de l'œuvre à la Renaissance, éd. A. Réach-Ngô, Paris, Classiques Garnier, 2014, p. 99-118, ici p. 101-102, n. 2).

10. C. Plinii Secundi Epistolae Familiares cum Barlandi Scholiis, Louvain, Thierry Martens, 1516 (Daxhelet, op. cit., ép. 20, p. 261-261). Voir aussi Daxhelet, op. cit., p. 192. Je traduis.

11. Sex comoediae, f. a1r (ép. 62, p. 319) : "ut sciant iuvenes quem fructum reportaturi sint ex comoediarum lectione.» (Toutes les traductions sont nôtres.)

12. Ibid., f. V2v (ép. 64, p. 320).

13. Ibid., f. A1v-A3r (ép. 65, p. 320-324).

14. À Paris, déjà, les étudiants disposaient de livres avec de larges marges et de grands interlignes, dépouillés de tout commentaire : ils pouvaient ainsi les annoter. Voir par exemple l'édition de l'Amphitryon de Plaute, parue à Paris chez Josse Bade ( $c f$. M. Ferrand, «Plaute à Paris. Diffusion et imitation des comédies plautiniennes au début du XvI $\mathrm{e}^{\mathrm{e}}$ siècle ", Paris, carrefour culturel autour de 1500, éd. O. Millet et L. A. Sanchi, [in] Cahiers V.L. Saulnier, 33, 2016, p. 189-203, ici p. 174-175).

15. Sex comoediae, f. A2r (ép. 65, p. 322). Voir aussi Daxhelet, op. cit., p. 77. 
16. De ratione studii (1512), trad. J.-C. Margolin dans Érasme, Éloge de la Folie. Adages. Colloques. Réflexions sur l'art, l'éducation, la religion, la guerre, la philosophie. Correspondance, éd. C. Blum, A. Godin, J.-C. Margolin, D. Ménager, Paris, Laffont, 1992, p. 460-461.

17. Cf. l'épître citée « Ad Lovanienses Tyrunculos » (Daxhelet, op. cit., ép. 64, p. 320).

18. Sex comoediae, f. A2r-v (ép. 65, p. 323).

19. Barlandus reprend notamment les propos d'Érasme dans le De ratione studii (op. cit., p. 444).

20. Tout cela se trouve chez Donat, De comoedia et tragoedia, éd. P. Wessner, Leipzig, Teubner, 1902- 1908, vol. I, p. 1, 1. 22. Ce texte est désormais attribué à Évanthius, comme l'indiquent B. Bureau, C. Nicolas et M. Ingarao dans leur présentation du site Hyperdonat (http:// hyperdonat.tge-adonis.fr).

21. Les savoirs collectés par d'autres se trouvent en revanche disséminés, parfois, dans le commentaire lui-même. C'est ainsi qu'il faut attendre le commentaire de L'Andrienne, IV, 5, pour lire une définition, reprise de Donat, des trois parties de l'intrigue : protasis, epitasis, catastrophe (Sex comoediae, f. L4r).

22. Ibid., f. A3v-A4v.

23. Ibid., f. a1r-a2v.

24. Ibid., f. A4v.

25. Sur la question du decorum personarum dans le commentaire de Barlandus, $c f$. E. W. Robbins, Dramatic characterization in Printed Commentaries on Terence (1473-1600), Urbana, The University of Illinois Press, 1951, p. 56-57. Le propos peut être complété par les analyses ponctuelles de M. T. Herrick. op.cit. Je ne reviens pas dans le présent article sur ce thème important des commentaires humanistes sur Térence.

26. Daxhelet, op.cit., p. 78-79. Dans le cadre du présent travail, je n'ai pas repris systématiquement cet examen. Sur l'entreprise éditoriale de la famille Estienne, voir M. Furno, op. cit., p. 102-103.

27. Sex comoediae, f. T1r. Cf. Daxhelet, op. cit., p. 24 et 79.

28. D'autres, au même moment, font un choix inverse, et insistent sur le geste philologique : voir M. Ferrand, art. cit.

29. Sex comoediae, f. V2v (ép. 63, p. 319).

30. Souvenir de saint Jérôme, Contra Rufinum, 1, 16: "Commentarii quid operis habent? Alterius dicta edisserunt, quae obscure scripta sunt plano sermone manifestant » (je souligne).

31. Sex comoediae, f. B4r.

32. À plusieurs reprises, il renvoie à son propre commentaire de l'Énéide (voir L'Andrienne, I, 3, v. 222, à propos de l'île d'Andros).

33. Sex comoediae, $\mathrm{f}$. B4r.

34. Je cite le commentaire de Donat et sa traduction d'après l'édition en ligne du site Hyperdonat.

35. Sex comoediae, f. B4r.

36. Ibid., f. L2v.

37. Sur l'emploi de la deuxième personne dans l'œuvre contemporaine de Josse Bade, voir Paul White, op. cit., p. 98 et F. Gonzalez Vega, « Para una tipologia del lector en el comentario literario de Jodoco Badio Ascensio (Silvae Morales, 1492)», Revista de estudios latinos 3, 2003, p. 155-168.

38. Voir la leçon, d'après Priscien, sur la construction des verbes pudet, taedet, piget (f. p3v) ou la longue digression de plus d'une page sur l'eclipsis dans la syntaxe latine (f. u3r-u4r).

39. Parmi les modernes, citons encore Pontano et Politien : le commentaire a aussi pour mérite de transmettre les savoirs élaborés par les Italiens, et d'opérer concrètement cette translatio studii qu'évoque la lettre liminaire à Jean Fevyn.

40. Sex comoediae, f. N3r-v.

41. À cet égard, et même si Barlandus n'utilise pas le terme, son commentaire relève bien du " commentum familiare » tel que le pratique Josse Bade, son compatriote et contemporain, à Paris ; voir les analyses de P. White, op. cit., p. 74-75: «Badius is thus not representative of the 'historicizing' 
tendency represented in the Italian philology of this period, whose object was in some sense to defamiliarize the past [...]. Badius's 'familiar' commentary, then, in the ways it was produced and used, had to do with the familiarization and domestication of texts. "

42. Sex comoediae, f. D4r (Andrienne, I, 4).

43. Ibid., f. Z4v (Les Adelphes, V, 4).

44. Ibid, f. C2r (L'Andrienne, I, 1).

45. Sur la distinction entre littera, sensus et sententia, dans la tradition du commentaire médiéval, voir par ex. J. Céard, «Les transformations du genre du commentaire ", L'Automne de la Renaissance (1580-1630), éd. J. Lafond et A. Stegmann, Paris, Vrin, 1981, p. 101-115 (ici, p. 102) : « la littera était l'explication grammaticale; le sensus la signification apparente des mots ; la sententia, l'intelligence profonde du texte."

46. M. T. Herrick, op. cit.

47. Sex comoediae, f. AA4v (Adelphes, V, 9). Il s'agit en fait de la Rhétorique à Herennius, IV, 39, dont il discute l'attribution à Cicéron au f. II3v.

48. P. White, op. cit., p. 62-64.

49. E. W. Robbins, op. cit., p. 57 : « leaning rather heavily upon Donatus, Barlandus comments more upon choice of words than upon larger events".

50. Ainsi, Barlandus ne reprend pas les remarques intéressantes de Donat sur l'exposition, dans la scène 1 de L'Andrienne. Cela ne l'empêche pas, toutefois, de donner lui-même un résumé précis de la situation.

51. Cf. Daxhelet, op. cit., p. 79-80.

52. Sex comoediae, f. C1r. Cf. Cicéron, De inventione, I, XxIII, 33.

53. Sex comoediae, f. C2v. Voir le texte complet d'Agricola, que Barlandus reproduit in extenso, dans Rodolphe Agricola, Écrits sur la dialectique et l'humanisme, éd. et trad. M. Van Der Poel, Paris, Champion, 1997, p. 173. Je reprends la traduction de M. Van der Poel.

54. Au folio N2v, Barlandus s'appuie encore sur Agricola pour rendre compte « de differentia poeticae dispositionis ab historia» (rubrique marginale : "sur ce qui différencie la dispositio de la poésie de celle de l'histoire ») : le philosophe y évoquait le dénouement de L'Andrienne. C'est la dispositio de la comédie elle-même qui est alors discutée.

55. Dialogi XLII per Hadrianum Barlandum, Cologne, Eucharius, 1530 (première édition : Louvain, Thierry Martens, 1524), f. D2r. Précisément, l'existence même du livre soulage la mémoire.

56. Sex comoediae, f. $01 \mathrm{v}-\mathrm{O} 2 \mathrm{v}$ : «Prologus Adriani Barlandis, non tamen usquequaque ad legem factus in Terentii Adelphos recitatus Lovanii quo tempore per eiusdem auditores magno studiosorum et civitatis concursu publice exhibita est haec Comoedia. ».

57. Sur les mises en scène du théâtre antique par A. Barlandus, voir E. Daxhelet, op. cit., p. 207-220, et M. Ferrand, " "Haud secus ac tradentes lampada cursu". Présence de l'Auctor dans les prologues de Martin Dorpius et Adrien Barlandus (Louvain, 1508-1516) », Écrire pour la scène (XVeXVIII ${ }^{e}$ siècle), éd. S. Chaouche, E. Doudet et O. Spina, [in] European Drama and Performance studies, 9, 2017 (à paraître).

58. Sex comoediae, f. H3r (Andrienne, III, 3).

59. Ibid., f. N4v (cf. le commentaire érasmien de l'adage I, 5, 45).

60. Ibid., f. E2v (Andrienne, I, 5).

61. Barlandus ne s'intéresse guère, en revanche, aux questions de métrique ou de prosodie. À l'occasion, il cite le Banquet poétique d'Érasme, donnant ainsi le schéma métrique du vers 66 de l' Andrienne.

62. Sex comoediae, f. NN3v.

63. Ibid., f. K3v.

64. Voir, dans l'Eunuque, I, 1, le f. O4v. Voir aussi la citation de Lorenzo Valla (In Antonium Raudensem, à propos des Adelphes, IV, 2), au f. U2v. 
65. À cet égard, ces formules ("si agenda est fabula», "qui recte volent agere fabulas », etc.) et le prologue original de Barlandus ont la même fonction que les illustrations dans certaines éditions contemporaines de Térence : elles rendent visible la théâtralité du texte.

66. Dialogi XLII, éd. cit., f. C6v.

67. Barlandus cite ici le commentaire érasmien de l'adage I, 2, 18, « Muti magistri », qui lui inspire en fait tout ce développement.

\section{AUTEUR}

\section{MATHIEU FERRAND}

Université Grenoble Alpes - UMR 5316 (Litt\&Arts/Translatio) 